\title{
EXTENSIONS OF RELATIVE TENSORS*
}

\author{
BY \\ OSWALD VEBLEN AND TRACY YERKES THOMAS†
}

1. Introduction. This paper is intended as an addendum to our previous paper on the Geometry of paths. formulas for the covariant derivatives and the higher extensions of relative tensors. These formulas are calculated by a process entirely analogous to that by which the corresponding formulas for ordinary tensors are obtained. Although the formulas are rather obvious the only one which we have observed in the literature is that for the covariant derivative of a relative tensor of the second order which was used by Einstein.§

2. Relative tensors. Let us denote by

$$
x^{i}=f^{i}\left(\bar{x}^{1}, \cdots, \bar{x}^{n}\right)
$$

an arbitrary analytic transformation of the coördinates $\left(x^{1}, \ldots, x^{n}\right)$ which can be written in the inverse form

$$
\bar{x}^{i}=g^{i}\left(x^{1}, \cdots, x^{n}\right) .
$$

Since the inverse transformation (2.2) exists, the jacobian of the transformation (2.1)

$$
\Delta=\left|\frac{\partial x}{\partial \bar{x}}\right|
$$

does not vanish identically. As we shall later require the derivative $\partial \Delta / \partial \bar{x}^{i}$ of the jacobian $\Delta$ we here note that it is given by

$$
\frac{\partial \Delta}{\partial \bar{x}^{i}}=\Delta \frac{\partial^{2} x^{\alpha}}{\partial \bar{x}^{i} \partial \bar{x}^{\beta}} \frac{\partial \bar{x}^{\beta}}{\partial x^{\alpha}} .
$$

- Presented to the Society, April 19, 1924.

$\dagger$ National Research Fellow in Mathematical Physics, University of Chicago.

† These Transactions, vol. 25 (1923), p. 551.

$\S$ A. Einstein, Zur allgemeinen Relativitätstheorie, Sitzungsberichte der Preu Bischen Akademie der Wissenschaften, 1923, p. 32. 
A set of functions $T_{i j \cdots k}^{l m \cdots n}$ will be said to form a relative tensor of weight $K$ if it transforms according to the equations

$$
\bar{T}_{i j \cdots k}^{l m \cdots n}=\Delta^{K} T_{\alpha \beta \cdots \gamma}^{\mu \nu \cdots \delta} \frac{\partial \bar{x}^{l}}{\partial x^{\mu}} \frac{\partial \bar{x}^{m}}{\partial x^{\nu}} \cdots \frac{\partial \bar{x}^{n}}{\partial x^{\delta}} \frac{\partial x^{\alpha}}{\partial \bar{x}^{i}} \frac{\partial x^{\beta}}{\partial \bar{x}^{j}} \cdots \frac{\partial x^{\gamma}}{\partial \bar{x}^{k}}
$$

when the variables are transformed by the equations (2.1). If $K=0, T_{i j \ldots k}^{l m \ldots n}$ is an ordinary tensor. In case $K=1, T_{i j \cdots k}^{l m \cdots n}$ is said to be a tensor density and is then usually written $\mathfrak{T}_{i j \cdots k}^{l m \ldots n}$. The justification of the name tensor density lies in the fact that the law of transformation of the integral

$$
\iint \cdots \int \mathfrak{T}_{i j \cdots k}^{l m \cdots n} d V
$$

extended over a definite $n$-dimensional region approaches more and more closely the law of transformation of an ordinary tensor $T_{i j \ldots k}^{l m \ldots n}$ as the region of integration closes down on a point. Thus the tensor density $\mathfrak{T}_{i j \cdots k}^{l m \cdots n}$ represents a sort of tensor $T_{i j \cdots k}^{l m \cdots n}$ of weight zero per unit of coördinate volume $d V$.

We easily see that the algebraic processes of addition, multiplication, and contraction of ordinary tensors will also hold for the case of relative tensors.

3. Covariant differentiation or first extension. Let the relative tensor $T_{i j \cdots k}^{l m \ldots n}$ of weight $K$ which is referred to the coördinates $\left(x^{1}, \cdots, x^{n}\right)$ be denoted by $t_{i j \cdots k}^{l m \cdots n}$ when referred to the system of normal coordinates $\left(y^{1}, \cdots, y^{n}\right)$ which are determined by the coordinates $\left(x^{1}, \cdots, x^{n}\right)$ and a point $\left(q^{1}, \cdots, q^{n}\right)$. We shall show that

$$
T_{i j \cdots k, p}^{l m \cdots n}=\left(\frac{\partial t_{i j \cdots k}^{l m \cdots n}}{\partial y^{p}}\right)_{0}^{l m}
$$

where the derivative is evaluated at the origin of normal coorrdinates, defines a set of functions $T_{i j \cdots k, p}^{l m \cdots n}$ of $\left(x^{1}, \cdots, x^{n}\right)$ which are the components of a relative tensor of weight $K$. The relative tensor $T_{i j \cdots \cdots, p}^{l m \cdots n}$ will be called the covariant derivative or first extension of the relative tensor $T_{i j \cdots k}^{l m \cdots n}$.

We denote by $\left(\bar{y}^{1}, \ldots, \bar{y}^{n}\right)$ the system of normal coördinates determined by the coordinates $\left(\bar{x}^{1}, \cdots, \bar{x}^{n}\right)$ and the point $\left(q^{1}, \cdots, q^{n}\right)$. Then

$$
\bar{y}^{i}=a_{\alpha}^{i} y^{\alpha}
$$


where the $a$ 's are constants. Furthermore denoting by $\bar{t}_{i j \cdots k}^{l m \ldots n}$ the relative tensor $T_{i j \cdots k}^{l m \cdots n}$ when referred to the normal coðrdinates $\left(\bar{y}^{1}, \cdots, \bar{y}^{n}\right)$ we have

$$
\bar{t}_{i j \cdots k}^{l m \cdots n}=\Delta^{K} t_{\alpha \beta \cdots \gamma}^{\mu \nu \cdots \delta} \frac{\partial \bar{y}^{l}}{\partial y^{\mu}} \frac{\partial \bar{y}^{m}}{\partial y^{\nu}} \cdots \frac{\partial \bar{y}^{n}}{\partial y^{\delta}} \frac{\partial y^{\alpha}}{\partial \bar{y}^{i}} \frac{\partial y^{\beta}}{\partial \bar{y}^{j}} \cdots \frac{\partial y^{\gamma}}{\partial \bar{y}^{k}} .
$$

In view of (3.2) the derivatives in (3.3) are constants and hence

$$
\text { (3.4) } \frac{\partial \bar{t}_{i j \cdots k}^{l m \cdots n}}{\partial \bar{y}^{p}}=\Delta^{K} \frac{\partial t_{\alpha \beta \cdots \gamma}^{\mu \nu \cdots \delta}}{\partial y^{\sigma}} \frac{\partial \bar{y}^{l}}{\partial y^{\mu}} \frac{\partial \bar{y}^{m}}{\partial y^{\nu}} \cdots \frac{\partial \bar{y}^{n}}{\partial y^{\delta}} \frac{\partial y^{\alpha}}{\partial \bar{y}^{i}} \frac{\partial y^{\beta}}{\partial \bar{y}^{j}} \cdots \frac{\partial y^{\gamma}}{\partial \bar{y}^{k}} \frac{\partial y^{\sigma}}{\partial \bar{y}^{p}} \text {. }
$$

Evaluating at the origin of normal coorrdinates we obtain

$$
\bar{T}_{i j \cdots k, p}^{l m \cdots n}=\Delta^{K} T_{\alpha \beta \cdots \gamma, \sigma}^{\mu \nu \cdots \delta} \frac{\partial \bar{x}^{l}}{\partial x^{\mu}} \frac{\partial \bar{x}^{m}}{\partial x^{\nu}} \cdots \frac{\partial \bar{x}^{n}}{\partial x^{\delta}} \frac{\partial x^{\alpha}}{\partial \bar{x}^{i}} \frac{\partial x^{\beta}}{\partial \bar{x}^{j}} \cdots \frac{\partial x^{\gamma}}{\partial \bar{x}^{k}} \frac{\partial x^{\sigma}}{\partial \bar{x}^{p}}
$$

which shows that the set of functions $T_{i j \cdots k, p}^{l m \cdots n}$ constitute a relative tensor of weight $K$ contravariant in the indices $(l, m, \cdots, n)$ and covariant in the indices $(i, j, \cdots, k, p)$.

We note that formulas analogous to those for the sum and product of two ordinary tensors likewise apply for the case of relative tensors.

To obtain the explicit formula involving the $\Gamma^{\prime}$ s and their derivatives for the relative tensor $T_{i j \cdots k, p}^{l m \cdots n}$ we make use of the equations

$$
t_{i j \cdots k}^{l m \cdots n}=\Delta^{K} T_{\alpha \beta \cdots \gamma}^{\mu \nu \cdots \delta} \frac{\partial y^{l}}{\partial x^{\mu}} \frac{\partial y^{m}}{\partial x^{\nu}} \cdots \frac{\partial y^{n}}{\partial x^{\delta}} \frac{\partial x^{\alpha}}{\partial y^{i}} \frac{\partial x^{\beta}}{\partial y^{j}} \cdots \frac{\partial x^{\gamma}}{\partial y^{k}} .
$$

Differentiating these equations and evaluating at the origin of normal coordinates we obtain

$$
\begin{aligned}
T_{i j \cdots k, p}^{l m \cdots n}=\frac{\partial T_{i j \cdots k}^{l m \cdots n}}{\partial x^{p}}+T_{i j \cdots k}^{\alpha m \cdots n} \Gamma_{\alpha p}^{l}+\cdots+T_{i j \cdots k}^{l m \cdots \alpha} \Gamma_{\alpha p}^{n} \\
\quad-T_{\alpha j \cdots k}^{l m \cdots n} \Gamma_{i p}^{\alpha}-\cdots-T_{i j \cdots \alpha}^{l m \cdots n} \Gamma_{k p}^{\alpha}-K T_{i j \cdots k}^{l m \cdots n} \Gamma_{\alpha p}^{\alpha} .
\end{aligned}
$$

This is the general formula of covariant differentiation. By using the tensors $D_{\alpha \tau \beta \ldots \nu}^{\sigma a b \ldots n}$ and $E_{\sigma a b \ldots n}^{\alpha \tau \beta \ldots \nu}$ (cf. The geometry of paths, loc. cit.) the form (3.7) may be abbreviated. Thus

$$
\begin{aligned}
T_{i j \cdots k, p}^{l m \cdots n}=\frac{\partial T_{i j \cdots k}^{l m \cdots n}}{\partial x^{p}}+\Gamma_{\sigma p}^{\tau} & T_{i j \cdots k}^{\alpha \beta \cdots \gamma} D_{\alpha \tau \beta \cdots \gamma}^{\sigma l m \cdots n} \\
& -\Gamma_{\tau p}^{\sigma} T_{\alpha \beta \cdots \gamma}^{l m \cdots n} E_{\sigma i j \cdots k}^{\alpha \tau \beta \cdots \gamma}-K T_{i j \cdots k}^{l m \cdots n} \Gamma_{\alpha p}^{\alpha} .
\end{aligned}
$$


4. Higher extensions. By a process similar to that employed for the covariant derivative we may show that

$$
T_{i j \cdots k, p \cdots q}^{l m \cdots n}=\left(\frac{\partial^{r} t_{i j \cdots k}^{l m \cdots n}}{\partial y^{p} \cdots \partial y^{q}}\right)_{0}
$$

defines a set of functions $T_{i j \cdots k, p \cdots q}^{l m \cdots n}$ of $\left(x^{1}, \ldots, x^{n}\right)$ which constitutes a relative tensor of weight $K$. The relative tensor $T_{i j \cdots k, p \cdots q}^{l m \cdots n}$ will be spoken of as the $r$ th extension of the relative tensor $T_{i j \cdots k}^{l m \cdots n}$ provided that there are $r$ indices $(p, \cdots, q)$. In case $r=1$ the extension reverts to the covariant derivative which we have already considered.

From its definition by means of the equations (4.1) we see that the extension $T_{i j \cdots k, p \ldots q}^{l m \cdots n}$ is symmetric in the indices $(p, \cdots, q)$. Thus

$$
T_{i j \cdots k, p \cdots q}^{l m \cdots n}=T_{i j \cdots k, u \cdots v}^{l m \cdots n}
$$

where $(u, \cdots, v)$ denotes any permutation of the indices $(p, \cdots, q)$.

The formulas for the extension of the sum and product of two relative tensors are similar to the corresponding formulas for the extension of the sum and product of two ordinary tensors.

General formulas of extension $(r>1)$ may be calculated by the same process as that employed in the calculation of the general formula of covariant differentiation (3.7). The formula for the $r$ th extension $T_{i j \ldots k, p q r \cdots u v}^{l m \ldots n}$ of a relative tensor $T_{i j \cdots k}^{l m \ldots n}$ of weight $K \neq 0$ involves the formulas for the first $r$ extensions of $T_{i j \cdots k}^{l m \ldots n}$ considered as a tensor of weight zero. For we have

$$
t_{i j \cdots k}^{l m \ldots n}=\Delta^{K} f_{i j \cdots k}^{l m \ldots n}
$$

where

$$
f_{i j \cdots k}^{l m \cdots n}=T_{\alpha \beta \cdots \gamma}^{\mu \nu \cdots \delta} \frac{\partial y^{l}}{\partial x^{\mu}} \frac{\partial y^{m}}{\partial x^{\nu}} \cdots \frac{\partial y^{n}}{\partial x^{\delta}} \frac{\partial x^{\alpha}}{\partial y^{i}} \frac{\partial x^{\beta}}{\partial y^{j}} \cdots \frac{\partial x^{\gamma}}{\partial y^{k}}
$$

Differentiating (4.3) and evaluating at the origin of normal coördinates

$$
\begin{aligned}
T_{i j \cdots k, p q r \cdots u v}^{l m \cdots n}=T_{i j \cdots k / p q r \cdots u v}^{l m \cdots n} & +S\left(\Delta_{p}^{K} T_{i j \cdots k / q r \cdots u v}^{l m \cdots n}\right) \\
& +S\left(\Delta_{p q}^{K} T_{i j \cdots k / r \cdots u v}^{l m \cdots n}\right)+\cdots \\
& +S\left(\Delta_{p q \cdots u}^{K} T_{i j \cdots k / v}^{l m \cdots n}\right)+\Delta_{p q r \cdots u v}^{K} T_{i j \cdots k}^{l m \cdots n}
\end{aligned}
$$


where

$$
\begin{aligned}
T_{i j \cdots k i p \cdots q}^{l m \cdots n} & =\left(\frac{\partial^{r} f_{i j \ldots k}^{l m \ldots n}}{\partial y^{p} \ldots \partial y^{q}}\right)_{0}, \\
\Delta_{p \cdots q}^{K} & =\left(\frac{\partial^{r} \Delta^{K}}{\partial y^{p} \cdots \partial y^{q}}\right)_{0},
\end{aligned}
$$

and $S(\quad)$ denotes the sum of the different terms obtainable from the one in parenthesis by forming arbitrary combinations of the subscripts $(p, q, r, \cdots, u, v)$. The expressions $T_{i j \cdots k / p \cdots q}^{l m \cdots n}$ are given by the ordinary formulas for the $r$ th extension of a tensor $T_{i j \ldots k}^{l m \ldots n}$ of weight zero, where $r$ is the number of indices $(p, \cdots, q)$, but these expressions do not in general constitute a tensor. The quantities $\Delta_{p \cdots q}^{K}$ have the values

$$
\begin{aligned}
& \Delta_{p}^{K}=-K \Gamma_{\alpha p}^{\alpha}, \\
& \Delta_{p q}^{K}=-K \Gamma_{\alpha p q}^{\alpha}-K \Gamma_{\beta p}^{\alpha} \Gamma_{\alpha q}^{\beta}+K^{2} \Gamma_{\alpha p}^{\alpha} \Gamma_{\beta q}^{\beta}, \\
& \begin{aligned}
\Delta_{p q r}^{K} & =-K \Gamma_{\alpha p q r}^{\alpha}-K S\left(\Gamma_{\beta p}^{\alpha} \Gamma_{\alpha q r}^{\beta}\right)-K S\left(\Gamma_{\beta p}^{\alpha} \Gamma_{\gamma q}^{\beta} \Gamma_{\alpha r}^{\gamma}\right) \\
& \quad+K^{2} S\left(\Gamma_{\alpha p}^{\alpha} \Gamma_{\beta q r}^{\beta}\right)+K^{2} S\left(\Gamma_{\alpha p}^{\alpha} \Gamma_{\beta q}^{\gamma} \Gamma_{\gamma r}^{\beta}\right)-K^{3} \Gamma_{\alpha p}^{\alpha} \Gamma_{\beta q}^{\beta} \Gamma_{\gamma r}^{\gamma},
\end{aligned}
\end{aligned}
$$

in which $\Gamma_{j k l}^{i}$ etc. are definite functions of the $\Gamma_{j k}^{i}$ and their derivatives (cf. The geometry of paths, loc. cit., p. 561). Thus we may write

$$
T_{i j \cdots k, p}^{l m \cdots n}=T_{i j \cdots k / p}^{l m \cdots n}-K T_{i j \cdots k}^{l m \cdots n} \Gamma_{\alpha p}^{\alpha}
$$

in place of the formula (3.7).

Any formula of extension of a relative tensor $T_{i j \cdots k}^{l m \cdots n}$ of weight $K$ may be obtained by substituting the proper values of $T_{i j \ldots k \mid p \ldots q}^{l m \ldots n}$ and $\Delta_{p \ldots q}^{K}$ in (4.4). A few particular expressions $T_{i j \ldots k / p \ldots q}^{l m \cdots n}$ are given in the Geometry of paths as formulas of extension of tensors of weight zero and these when substituted into the equations (4.4) together with the above values of $\Delta_{p \ldots q}^{K}$ give complete formulas in terms of the $\Gamma_{j k}^{i}$ and their derivatives for the extensions of certain relative tensors of weight $K$. 